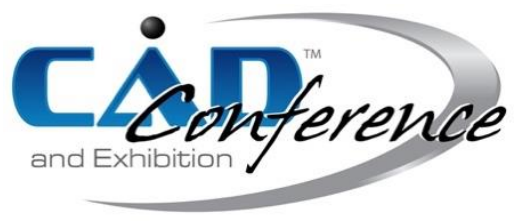

Title:

The Kandinsky Experience: A Multisensory AR Application for Cultural Heritage

Authors:

Sara Belloli, sara1.belloli@mail.polimi.it, Politecnico di Milano

Sara Porro, sara.porro@mail.polimi.it, Politecnico di Milano

Vikram Singh Virk, vikramsingh.virk@mail.polimi.it, Politecnico di Milano

Roberta Etzi, roberta.etzi@polimi.it, Politecnico di Milano

Alberto Gallace, alberto.gallace1@unimib.it, Mind and Behavior Technological Center, Università di Milano Bicocca

Monica Bordegoni, monica.bordegoni@polimi.it, Politecnico di Milano

Marina Carulli, marina.carulli@polimi.it, Politecnico di Milano

Keywords:

Augmented Reality, Multisensory Perception, User Experience, Cultural Heritage

DOI: 10.14733/cadconfP.2020.268-272

Introduction:

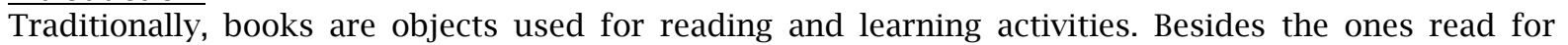
pleasure, books are used for educational purposes and for improving readers' knowledge about specific topics. In recent years the widespread of ICT technologies in everyday life has also had an impact on books, which have been subjected to a radical transformation. Instead of ink and paper, today digital contents are displayed on various devices, such as e-book readers, computer displays, tablets, and smartphones. This revolution, which has been embraced with scepticism by some "purist" users, is based on a change of the support, from physical to digital. Consequently, readers (especially younger readers) are trying to adapt to these new technologies and change their reading behaviors in order to exploit the digital potential [13].

However, even though today technological devices used for reading e-books (tablets, e-readers etc.) are still poorly exploited, they can represent a fundamental tool to make the reading experience more immersive by using a complete multisensory approach, in which more senses are stimulated in a unique stream. Moreover, digital books lack somehow of the multisensory appeal of traditional book, something that might lead to a reduction in learning performance and awareness (see [5], for a recent metanalysis where digital and traditional reading is compared).

Indeed, the possibility to use a digital support opened new possibilities such as, from the addition of sounds and audio tracks, or the interaction through touch displays to a transformation into Virtual (VR) and Augmented Reality (AR) applications. Specifically, three trends and directions of book evolution have been identified by Park et al. [15]. These are digitalization, augmentation and hypermediation. Moreover, they suggested three research and business directions: open hypermediation, e-book augmentation, and mobile-device based augmentation [1]. These approaches are strictly related to traditional interactive books, which require the active involvement of the reader. Interactive books are usually created for children, in order to capture their attention. In these books the interaction can be usually based on touch (by using different textures, moving parts, and so on), audio (with musical pages), smell (with the so-called "scratch and sniff" stickers). Stimuli from these senses are typically integrated with illustrations and text. Consequently, interactive books are intrinsically multisensory in their nature.

Augmented and multisensory approaches have been proven to be effective both in the case of books for children and young people and in the case of adults. Children and young people can particularly 
take advantage of augmented multisensory approaches, allowing them to keep their attention high [1, 18].

The primary objective of the use of these technologies is to make the reading and learning activities much more engaging, immersive, efficient and suitable for different types of readers. In particular, the common idea is to "extend" the target audience also to those who are not experts in the topics described in the books who, through the new co-communication methods, can better understand complex concepts. In this sense, in fact, the possibility of viewing the history of the pieces of art and their meaning instead of only reading or listening to the explanation, or rather of looking at images / animations instead of reading texts, reduces the need to have experienced and knowledgeable users, and increases the possibilities of learning and remembering information. This also comply with the wellestablished 'dual code theory of learning' in human cognition, suggesting that learning is more efficient when information is not presented only via a single sensory channel (e.g. [4]). In the "non-expert users" category, it is also possible to include children, who are often fascinated by science, nature and art, but are hardly interested in static representations that are very difficult to understand. Moreover, nowadays the user experience is one of the fundamental features of books and is strictly linked to the user - device (book) interaction at physical, perceptual and cognitive levels. This means that it includes also the design of multisensory experiences that also satisfy sight, touch, hearing and smell senses to improve the user's engagement. This is related to the fact that typically the human brain integrates information from different sensory modalities [17] in order to define a univocal final experience of the external world. Psychological and neuroscientific research has shown that perception is always multisensory in nature: whenever we interact with the external world our brain integrates pieces of information from different sensory modalities as a function of certain binding principles [17]. In particular, the sense of smell plays an important role, since it recalls memories and deep emotions both at conscious and unconscious level. Thanks to these effects on humans, smells can help the customer to feel involved into and emotionally linked with artworks. In human history odors have represented important elements of culture and religions.

In the last years, several studies have focused on the integration of odors in VR and AR environments by developing devices named Olfactory Displays, based on several technologies, for different applications [3, 14]. Even if olfactory display devices are still evolving and some technical issues still remain unsolved, some companies have developed olfactory displays for VR/AR applications. Recently, some new devices have been launched on the market. For example, the Vaqso device (https://vaqso.com/), the Olorama scents generator (https://www.olorama.com/professional-scentgenerators/), and the Feelreal device (https://feelreal.com/). However, the use of the sense of smell is underexplored, especially when its possible applications and impact can be quite effective. Indeed, the sense of smell is one of the most evolutionary ancient sense in humans and animals, and it is linked to visceral emotions in comparison to the other senses. Because of that, odors can have an impact also on implicit (unconscious) processing of information, thus affecting our feelings, moods, memories, and so on. In [6], it has been proven that odours can improve reading activities and make the reading experience more immersive and can increase the level of concentration and the learning performances of people.

The purpose of the research work presented in this paper is to design and develop an interactive multisensory AR application (based on sight, hearing, and smell and senses), which can be used for improving the user's experiences of art books, and the comprehension of their contents. Specifically, the paper presents a case study where a multisensory AR book concerning the Kandinsky's artworks has been designed and developed. Then, preliminary testing sessions with users concerning the users' involvement, comprehension and overall experience have been carried out, and preliminary analyses of the collected data will be presented.

\section{Main idea}

The Kandinsky experience is a multisensory AR experience that involves sight, hearing and smell senses and aims at improving the users' engagement and understating of the content of the Kandinsky's work.

Specifically, the aim of the Kandinsky experience was augmenting the experience of the user of a journey throughout the Kandinsky' work by using an AR application integrated with audio and olfactory stimuli for allowing him/her to be more immersed in the subject. 
So, the Kandinsky experience has the aim to provide the users with a new and different way of comprehension and enjoyment of art. Thanks to the simultaneous presentation of the specificallydeveloped AR contents and the olfactory stimuli, when the users appreciate the artworks, their feelings and emotions are amplified as result of the sensory integration. Moreover, using AR technology and olfactory devices to solicit visual and olfactory experiences increases the chances of generating longlasting memories in the users' mind. In fact, smell plays a crucial role in creating and retrieving emotional memories, thanks to the direct link between the olfactory cortex and the limbic system [8].

The AR multisensory experience is based on a deep analysis of the Kandinsky's work and his theories about colors, shapes, lines, sounds and the synesthetic relationship between them, as reported in $[8,9]$. Indeed, the Kandinsky's approach to art is intrinsically experimental and multisensory, and it is based on the idea that every element that is part of an artistic composition arouses a specific vibration in the soul of the viewer and that the combination of these stimuli generates a unique emotional experience. This vision of art is not just limited to pictorial works, but embraces the overall sensorial realm, including the auditory and the olfactory stimuli.

Consequently, the experience has been designed to illustrate Kandinsky's theories through some of his artworks organized in three main sections, which are the Colors, the Lines, and the Shapes [10, 11]. In these main sections, the relationship between the pictorial element and the auditory stimuli are enhanced respecting the author indications, while the corresponding relation with olfactory stimuli, that was just suggested by Kandinsky, has been reinterpreted. The associations between scents and paintings have been chosen according to the characteristics of each visual element described by Kandinsky combined with the analysis of the state of the art concerning cross modal associations $[2,6,7,9,12,16]$ and the result of a preliminary test run specifically for this purpose, focusing on the association between specific olfactory stimuli, auditory and visual stimuli.

For what concerns the technological development of the AR multisensory Kandinsky experience, it consists of three main components.

The first main component consists of the AR application displayed on tablets or smartphones, which allows animating the most representative paintings of Kandinsky. For example, framing the painting entitled Several Circles (Fig.1a), users can see the flat representation of the painting coming alive, acquiring the third dimension and reproducing the movement effect caused by the color applied on the shape (Fig. 1b).
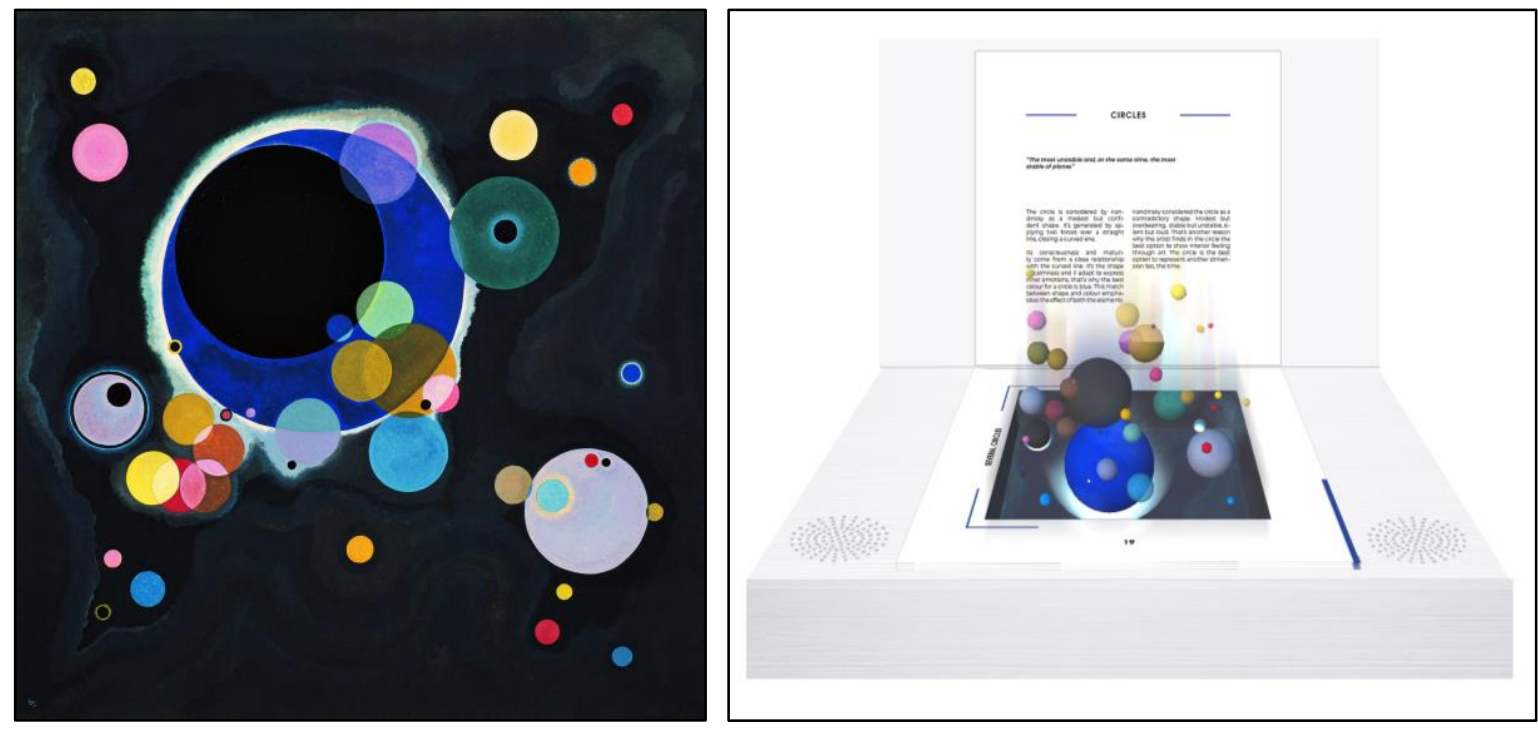

Fig. 1: (a) Several Circles by Kandinsky (b) Several Circles animated through the AR multisensory Kandinsky experience. 
When triggered, the AR application also provides two more sensorial stimuli associated to the Kandinsky's work. For example, the Blue Painting by Kandinsky has been matched to the "8th Symphony" of Shostakovich and to the scent of Sandal, creating an evocative AR animation in which movement, music and scent are combined to enhance the characteristics of the color blue, as described by Kandinsky. A second modality has been implemented, allowing the user to explore the 3D model of the painting. In the meanwhile, a recorded voice describes the characteristics of the main elements characterizing each painting. In the $\mathrm{AR}$ application each paint is used as marker to trigger the application. The Unity3D (https://unity.com/) and the Vuforia (https://developer.vuforia.com/) software tools have been used to develop the AR application. Specifically, the animations of the paintings have been developed in Unity3D, and the Vuforia software has been used for the development of the AR visualization of the contents.

The second main component of the system consists of the case made to accommodate the book and to place the olfactory displays that deliver the odors. Each olfactory display consists of a fan conveying air through a cartridge soaked with some drops of liquid scents. The case is provided with two olfactory displays placed on two sides of the niche created for the book, to be able to provide a combination of two scents as olfactory stimuli when needed. Due to the fact that each paint corresponds to a specific olfactory stimulus, the cartridges can be easily replaced when indicated by the AR application. The odors to be released have been carefully selected to create an immersive experience and having an emotional link with the paint. More, the final aim of the use of the application is to augment the comprehension of the Kandinsky's work and its meaning. The AR application is connected to an Arduino Uno board equipped with a Bluetooth module for allowing the communication among the application, the board and the olfactory devices.

The third component of the system is the main book containing a brief description of Kandinsky's theory divided into nine chapters and associated with nine selected paintings. Each paint has been used as marker for the AR application. The Architecture of the system is shown in Fig. 2.

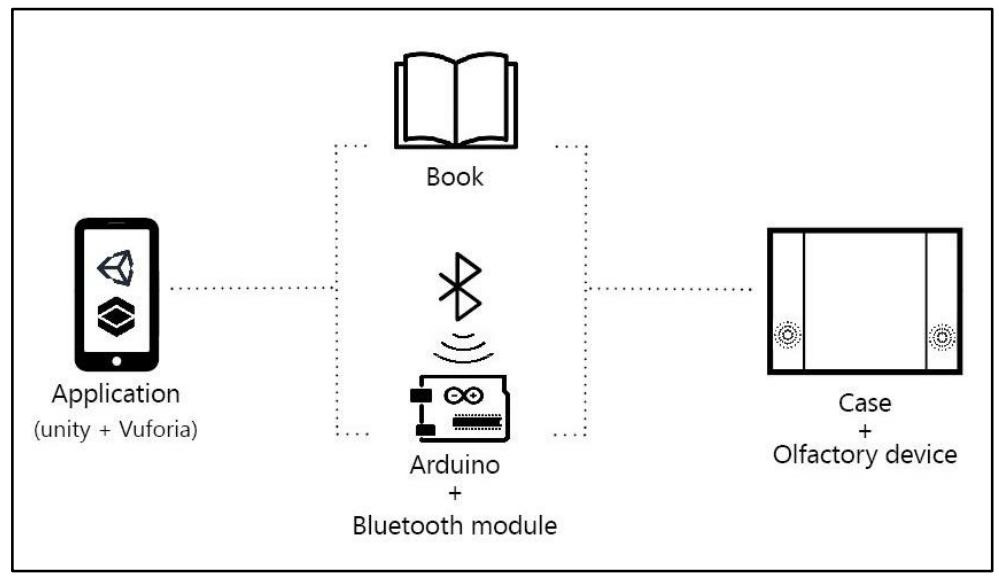

Fig. 2: The architecture of Kandinsky - Experience Book system.

In order to interact with the system, users are asked to open the AR application on their personal smartphones or tablets and frame one of the paints depicted in the book. Consequently, the AR application automatically recognizes the paint and displays the corresponding animation, music, odor and oral description.

\section{Conclusions}

The research work presented in this paper focuses on the possibility to improve the user experience of artworks books through a multisensory AR application (based on sight, hearing, and smell). 
Specifically, the Kandinsky application has been designed and developed with the aim of improving the user's engagement and understanding of the content. Particular attention has been devoted to the adoption of olfactory stimuli and their integration with the other senses. Indeed, the olfactory stimuli have been designed with the aim of impacting on the subconscious and conscious levels of users to evoke emotions and generate long-lasting memories. So, the preliminary testing sessions carried out with users concerned the users' perceptual, emotional and cognitive reactions to the overall experience, as well as the usability of the interactive system. In the final paper a preliminary analysis of the collected data will be presented.

It is worth saying that both the use of Augmented Reality and the multisensory approach are still rarely used for innovative books. On the opposite, these technologies can be effectively used to create an emotional link between the readers and artworks, to improve the users' experience and knowledge. These applications indeed can be exploited as learning tools to be applied to a huge variety of topics and then have a powerful role from an educational point of view.

So, in an envisaged scenario, multisensory AR applications similar to those presented can be developed and implemented in a wide range of books providing as the base product the case containing the olfactory device, designed to be able to host different books and applications.

\section{References:}

[1] Alam, K.-M.; Rahman, A.-S.; El Saddik, A.: Mobile haptic e-book system to support 3D immersive reading in ubiquitous environments. ACM Trans. Multimedia Comput. Commun. Appl., 9(4), 2013

[2] Blazhenkova, O.; Kumar M. M.: Angular Versus Curved Shapes: Correspondences and Emotional Processing, Perception., 47(1), 2018, 67-89, https://doi.org/10.1177/0301006617731048

[3] Bordegoni, M.; Carulli, M.; Shi, Y.; Ruscio, D.: Investigating the effects of odour integration in reading and learning experiences, Interaction Design and Architecture(s), 32, 2017, 104-125.

[4] Brunye, T.-T.; Taylor, H.-A.; Rapp, D.-N.: Repetition and dual coding in procedural multimedia presentations. Applied Cognitive Psychology, 22, 2008, 877-895.

[5] Clinton, V.: Reading from paper compared to screens: A systematic review and meta-analysis, Journal of Research in Reading, 42 (2), 2019, 288-325, https://doi.org/10.1111/1467-9817.12269.

[6] Deroy. O.; Crisinel, A-S.; Spence, C.: Crossmodal correspondences between odors and contingent features: odors, musical notes, and geometrical shapes, Psychon Bull Rev., 20(5), 2013, 878-96. https://doi.org/10.3758/s13423-013-0397-0

[7] Hanson-Vaux, G.; Crisinel, A-S.; Spence C.: Smelling Shapes: Crossmodal Correspondences Between Odors and Shapes, Chem Senses, 38(2), 2013, 161-166. https://doi.org/10.1093/chemse/bjs087

[8] Herz, R.: The role of odor-evoked memory in psychological and physiological health. Brain sciences, 6(3), 2016, 22.

[9] Kaeppler, K.; Crossmodal Associations Between Olfaction and Vision: Color and Shape Visualizations of Odors, Chemosensory Perception, 11(2), 2018, 95 - 111.

[10] Kandinsky, W.: Lo spirituale nell'arte, edited by Elena Pontiggia, SE, Milano, 2005.

[11] Kandinsky, W.: Punto, linea, superficie: contributo all'analisi degli elementi pittorici. Milano: Adelphi, 1968.

[12] Levitan, C.-A.; Charney, S.-A.; Schloss, K.-B.; Palmer, S.-E.: The Smell of Jazz: Crossmodal Correspondences Between Music, Odor, and Emotion, CogSci 2015.

[13] Liu, Z.: Reading behavior in the digital environment, Journal of Documentation, 61(6), 2005, 700 712.

[14] Nakamoto, T., Human Olfactory Displays and Interfaces: Odor Sensing and Presentation, Information Science reference, 2013.

[15] Park, A.; Lee K.-J.; Casalegno, F.: The three dimensions of book evolution in ubiquitous computing age: Digitalization, augmentation, and hypermediation. In Proceedings of the IEEE International Conference on Sensor Networks, Ubiquitous, and Trustworthy Computing, 2010, 374-378.

[16] Smith, B. C.: Human Olfaction, Crossmodal Perception, and Consciousness. Chemical Senses, 42(9), 2017, 793-795, https://doi.org/10.1093/chemse/bjx061.

[17] Spence, C.; Driver, J.: Crossmodal space and crossmodal attention. Oxford University Press, 2004

[18] Spence, C.; Gallace, A.: Multisensory design: Reaching out to touch the consumer, Psychology \& Marketing, 28, 2011, 267-308. 